\title{
LIMITES ÉTICOS E CONTRIBUIÇÕES PARA A SOCIEDADE: UMA ANÁLISE DAS IMPLICAÇÕES DA APROXIMAÇÃO ENTRE NEUROCIÊNCIA E COMPORTAMENTO DO CONSUMIDOR
}

\section{ETHICAL LIMITS AND CONTRIBUTIONS TO SOCIETY: ANALYZING THE IMPLICATIONS OF THE CONNECTIONS BETWEEN NEUROSCIENCE AND CONSUMER BEHAVIOR}

Stefânia Ordovás de Almeida Pontifícia Universidade Católica do Rio Grande do Sul - PUC-RS stefania.almeida@pucrs.br

Vinícius Sittoni Brasil Pontifícia Universidade Católica do Rio Grande do Sul - PUC-RS vinicius@pucrs.br

Lélis Balestrin Espartel Pontifícia Universidade Católica do Rio Grande do Sul - PUC-RS $\underline{\text { lbespartel@pucrs.br }}$

Fernanda Catelan Callegaro Pontifícia Universidade Católica do Rio Grande do Sul - PUC-RS fercallegaro@hotmail.com

Submissão: $31 / 03 / 2015$

Aprovação: 17/08/2015 


\section{RESUMO}

Desde explicações baseadas na racionalidade econômica até a busca do entendimento profundo da forma de pensar e agir, pesquisadores buscam métodos que permitam melhor compreender o consumidor. A despeito das evidências quanto às contribuições que a neurociência pode trazer ao entendimento de fenômenos de consumo, emergem discussões com distintos prismas na literatura, tendo em vista seu potencial impacto na sociedade. Os limites da ética em estudos desta natureza e a apropriação e uso dos achados resultantes destes estudos pela área de marketing permeiam expressivo debate. Se de um lado há forte inclinação junto a alguns autores em aceitar os benefícios da neurociência do consumo, outros ponderam sobre limites de seu uso e sobre a real necessidade de serem utilizadas técnicas neurocientíficas em marketing. Diante do crescente interesse pelo tema e de inúmeras críticas ao potencial manipulador do marketing, a partir de uma revisão da literatura e de entrevistas de profundidade com especialistas, este artigo analisa o estado da arte da interface entre neurociência e consumo e suas implicações à pesquisa em marketing e à sociedade. Os resultados apontam que, ao menos no atual contexto, as técnicas neurocientíficas devem ser utilizadas com cautela e estão longe de representar a resposta final buscada pelos pesquisadores da área.

Palavras-chave: Neurociência do consumo. Neuromarketing. Comportamento do consumidor. Ética na pesquisa. 


\begin{abstract}
Starting on explanations based on economical rationality up to the deep understanding of how we think and act, researchers search for methods that allow for a better understanding of the consumer. Despite evidences regarding the contributions that neuroscience can bring to consumer research, discussions emerge regarding different views on the literature, regarding its potential impact on society as a whole. The ethical limits of this nature and the appropriation and use of the findings of these studies by the Marketing area permeate relevant debates. On the one hand some authors agree that it's best to accept the benefits of consumer neuroscience, while others ponder about the limits of its use and even on the real need to use neuroscientific techniques in Marketing. In view of the growing interest in this subject and numerous criticisms of the manipulative potential of Marketing, based on two methodological approaches (literature review and in-depth interviews), this article analyzes the state of the art interface between neuroscience and consumption and its implications and possible contributions to research in marketing and society. The results show that, at least in the present context, the neuroscientific techniques should be used with caution and are far from representing the final answer sought by researchers.
\end{abstract}

KeyWords: Consumer neuroscience. Neuromarketing. Consumer behavior. Ethics. 


\section{Introdução}

$\mathrm{Na}$ medida em que as atividades de consumo passaram a representar importante faceta da sociedade e, de modo específico, da expressão dos indivíduos, o marketing depara-se com questionamentos acerca de seu papel. Na pauta das discussões estão temas diversos tais como responsabilidades no estímulo de um consumo excessivo e nocivo ou a inobservância de limites individuais na busca de informações cada vez mais precisas quanto ao comportamento humano. Recentemente, com o advento de técnicas neurocientíficas avançadas, as discussões sobre sua utilização pelo marketing, seus limites éticos e seus impactos na sociedade tornaram-se relevantes.

Desde o enfoque da racionalidade econômica até a busca pela compreensão dos processos cognitivos e emocionais subjacentes à tomada de decisão, o estudo do comportamento do consumidor busca compreender a complexidade existente nas decisões de consumo. A Neurociência do Consumidor estuda "as condições e processos neurais que fundamentam o consumo, o seu significado psicológico e as suas consequências comportamentais" (REIMANN et al., 2011, p. 610). Mais especificamente, a aplicação das técnicas neurocientíficas para compreender fenômenos de consumo podem gerar dados sobre os processos subjacentes (cognitivos-afetivos) que guiam os comportamentos de consumo observáveis. Por exemplo, como escolhas de consumo, aparentemente semelhantes, podem ser orientadas por trajetos neurais distintos (VENKATRAMAN; PAYNE, 2011; SOLNAIS et $a l ., 2013)$. Como resultado, as técnicas neurocientíficas vêm sendo consideradas para ampliar a compreensão da dinâmica que permeia o modelo clássico - estímulo do ambiente-resposta comportamental, uma vez que podem permitir a análise aprofundada dos aspectos inobserváveis (black box), que norteiam essa relação (FUGATE, 2007; ENGEL; KOLLAT; BLACKWELL, 1968).

Entretanto, com a evolução destes estudos, as terminologias Neurociência do Consumidor e Neuromarketing têm gerado dúvidas no que tange à sua compreensão e distinção. Neste sentido, os autores Hubert e Kenning (2008) chamam a atenção para os aspectos que tornam estes termos distintos na literatura acadêmica e no âmbito gerencial. Neuromarketing é conceituado por Lee, Broderick e Chamberlain (2007, p. 200) como "a aplicação dos métodos de pesquisa da Neurociência para analisar e entender o comportamento humano em relação aos mercados e as trocas de Marketing”. Hubert (2010), por sua vez, destaca que o termo Neurociência do Consumidor é mais apropriado para se referir ao fenômeno na pesquisa acadêmica. Sob esse enfoque, a Neurociência do Consumidor 
relaciona-se à compreensão científica do fenômeno, enquanto o termo Neuromarketing consiste na aplicação prática do conhecimento consolidado na pesquisa acadêmica para o contexto empresarial (HUBERT, 2010).

Nessa linha de pesquisas, dois journals devotaram números especiais para tratar de temas que interligam a neurociência ao marketing. O primeiro deles foi o Journal of Consumer Behaviour, na sua edição 4-5 de 2008. Dada a incipiência da temática, estes artigos variavam desde uma revisão sobre os estudos até então realizados (SENIOR; LEE, 2008), até discussões sobre frameworks teóricos para guiar a agenda de pesquisas sobre o tema (GARCIA; SAAD, 2008), dentre outros apresentando resultados de pesquisas utilizando técnicas neurocientíficas. A segunda publicação a trazer uma edição temática foi o Journal of Consumer Psychology, no seu número 22 de 2012, cujo escopo se assemelha ao JCB, explorando diferentes facetas desse novo campo de estudos. No campo do comportamento do consumidor, o artigo publicado por Solnais et al. (2013), no Journal of Economic Psychology, dedicou-se a analisar, em profundidade, as principais esferas e/ou temáticas de contribuições do campo Neurociência do consumidor na pesquisa em Marketing. No contexto brasileiro, os trabalhos de Oliveira, Giraldi e Santos (2014) e Santos et al. (2014) apresentam uma extensa revisão da literatura sobre o tema.

A despeito destes avanços e discussões na temática pesquisada, como toda área em ascensão e revestida de um caráter de novidade, surgem inúmeras questões a serem respondidas. Por exemplo, as ferramentas da neurociência permitirão a compreensão definitiva de como se processam as decisões de consumo? É possível, de fato, manipular as decisões do indivíduo a partir da compreensão do funcionamento do cérebro? Quais são as implicações éticas para a pesquisa em comportamento do consumidor? E quais aplicações dos achados de estudos em neurociências e consumo podem reverter em benefícios para a sociedade?

Diante de tais questionamentos, o presente artigo tem como objetivo central a discussão da neurociência aplicada ao consumo a partir da convergência de uma revisão da literatura sobre o tema e uma série de entrevistas de profundidade realizadas com especialistas e pesquisadores. Exploram-se as implicações éticas deste tipo de pesquisa e seus possíveis riscos e contribuições à sociedade, ampliando a forma de pensar tais contribuições para muito além do denegrido conceito de "buy bottom". Estruturalmente, os resultados deste artigo são divididos em três momentos: a) métodos e contribuições da neurociência para o entendimento do consumidor; b) questões éticas relacionadas à pesquisa; e c) possibilidades de pesquisa e 
interesses para a sociedade. Finalizando, são elencadas algumas considerações finais e futuros desdobramentos.

\section{Procedimentos metodológicos}

Considerando-se o objetivo proposto neste estudo, julgou-se apropriado o emprego do método qualitativo, dado o estudo ser de natureza exploratória (MALHOTRA, 2008). Os procedimentos metodológicos utilizados convergem os achados resultantes de uma revisão da literatura pertinente ao tema, acrescido dos resultados de uma coleta de dados primários de natureza qualitativa. Tal opção deve-se, principalmente, ao fato de haver reduzida produção brasileira sobre o tema, sendo esta concentrada em artigos de cunho teórico.

Empregou-se como técnica de coleta de dados a entrevista em profundidade, através da pré-elaboração de um roteiro de entrevistas semiestruturado, gerado com o suporte da revisão da literatura. Posteriormente, o roteiro foi validado por especialistas da área (FLICK, 2009). A fim de obter dados mais aprofundados e que fossem ao encontro do objetivo de pesquisa proposto, utilizaram-se alguns critérios para a seleção dos especialistas entrevistados, que foi constituído por dois grupos: comunidade acadêmica de Marketing e Neurocientífica.

Primeiramente, realizou-se uma listagem contendo vinte e quatro potenciais especialistas, isto é, profissionais que possuem expressividade na comunidade acadêmica em vista às pesquisas concretizadas nas áreas de Marketing e/ou Neurociências e que, sobretudo, refletem o domínio teórico e metodológico acerca do tema proposto neste estudo. Portanto, esses especialistas foram considerados aptos para fornecerem percepções significativas sobre o fenômeno investigado. Para refinar essa seleção, também foi realizada uma checagem em relação ao nível das instituições de ensino as quais os potenciais especialistas estão vinculados. Dessa forma, no contexto da pesquisa nacional, privilegiaram-se os especialistas que pertencem aos principais programas de pós-graduação em Administração e Neurociências com conceito superior a 4 pela CAPES. Visto que o grupo também foi estabelecido por pesquisadores internacionais, checou-se se os mesmos integram os programas de pósgraduação das 200 melhores universidades do mundo, conforme classificação proposta pelo ranking Times Higher Education. O segundo critério utilizado incidiu sobre a produção acadêmica dos potenciais especialistas selecionados. Em relação ao contexto de pesquisa nacional, checou-se se os mesmos possuem um histórico de publicação em revistas e/ou periódicos classificados entre A1 e B2, segundo o critério Qualis. Em contrapartida, no 
contexto internacional, checou-se se as publicações dos potenciais especialistas estavam nos considerados Top Journals na área de Marketing, Medicina e/ou Neurociências, ou seja, com fator de impacto expressivo.

Depois de estabelecidos esses critérios e posterior checagem, dezesseis especialistas demonstraram interesse em participar do estudo, cujo convite foi realizado via e-mail eletrônico e/ou mediante pré-agendamento face a face e/ou troca de contatos em eventos e congressos da área. Antes de iniciar a coleta de dados foi realizada uma entrevista pré-teste, que possibilitou a verificação quanto à compreensão dos questionamentos elaborados. Essa etapa também contribuiu para que pequenos ajustes de terminologia nos roteiros de entrevistas fossem concretizados (por exemplo, adequação de termos técnicos utilizados no campo da Neurociência e/ou Medicina). Não houve modificações no roteiro além destes pequenos ajustes de terminologia, de forma que os relatos oriundos da entrevista pré-teste foram incluídos em posterior análise, em vista à riqueza dos dados resultantes, da homogeneidade dos roteiros de aplicação e da escassez de entrevistados qualificados com o perfil pesquisado. Após essa etapa, sucederam-se as demais entrevistas em profundidade, sendo que apenas treze especialistas vieram a participar de fato do presente estudo. Das treze entrevistas em profundidade, quatro foram realizadas presencialmente, enquanto nove foram realizadas online, utilizando como recurso o software de conversação Skype.

Segundo Flick (2009), as entrevistas em profundidade podem ser transferidas do ambiente real para o ambiente virtual, sem que haja comprometimento no processo de obtenção dos dados. O Quadro 1 sintetiza o perfil dos especialistas que integraram esse estudo. Os especialistas que participaram do estudo foram identificados, com o devido consentimento dos mesmos; porém, cabe destacar que no processo de análise dos dados os mesmos não serão revelados. Optou-se por manter a sua identificação em sigilo, a fim de zelar por questões éticas de pesquisa. Destaca-se, também, que na apresentação dos resultados a identificação numérica de cada entrevistado não está relacionada com a ordem de citação abaixo.

Quadro 1

Perfil dos especialistas entrevistados

\begin{tabular}{|c|c|c|}
\hline Especialista & Interesses de pesquisa & Instituição \\
\hline Walter Nique & $\begin{array}{l}\text { Satisfação do Consumidor, Valores e Comparação de } \\
\text { Culturas. }\end{array}$ & $\begin{array}{l}\text { Universidade Federal do Rio } \\
\text { Grande do Sul - UFRGS. }\end{array}$ \\
\hline José Hernandez & Inferências no Processo de Decisão do Consumidor. & Centro Universitário da FEI. \\
\hline Kenny Basso & $\begin{array}{l}\text { Confiança, Violação e Recuperação da Confiança; } \\
\text { Falhas e Recuperação de Serviço e Influências } \\
\text { Ambientais no Comportamento de Consumo. }\end{array}$ & Faculdade Meridional - IMED. \\
\hline
\end{tabular}




\begin{tabular}{|c|c|c|}
\hline Vinícius Brei & $\begin{array}{c}\text { Adaptação/Saciação/Habituação; Desejos de Consumo } \\
\text { e Insaciabilidade. }\end{array}$ & $\begin{array}{l}\text { Universidade Federal do Rio } \\
\text { Grande do Sul - UFRGS. }\end{array}$ \\
\hline $\begin{array}{l}\text { Paulo Henrique } \\
\text { Muller Prado }\end{array}$ & $\begin{array}{c}\text { Satisfação, Qualidade e Lealdade; Estruturas } \\
\text { Cognitivas e Adoção de Inovações; Relacionamento } \\
\text { Consumidor-Marca. }\end{array}$ & $\begin{array}{l}\text { Universidade Federal do Paraná - } \\
\text { UFPR. }\end{array}$ \\
\hline Eduardo Andrade & Respostas Emocionais do Consumidor. & $\begin{array}{c}\text { Fundação Getulio Vargas do Rio } \\
\text { de Janeiro - FGV-RJ. }\end{array}$ \\
\hline Juliano Laran & $\begin{array}{c}\text { Propriedades Cognitivas dos Objetos; Autorregulação; } \\
\text { Aprendizagem Associativa. }\end{array}$ & University of Miami. \\
\hline $\begin{array}{l}\text { Marcus Cunha } \\
\text { Junior }\end{array}$ & $\begin{array}{c}\text { Aprendizado; Memória; Processamento de } \\
\text { Informações. }\end{array}$ & University of Georgia - UGA. \\
\hline Giuliana Isabella & Neurociência do Consumidor; Neurociência Cognitiva. & $\begin{array}{l}\text { Universidade Federal de São } \\
\text { Paulo - USP. }\end{array}$ \\
\hline Antoine Bechara & $\begin{array}{c}\text { Neurologia e Neuropsicologia de Comportamentos } \\
\text { Complexos; Tomada de Decisão; Vícios e } \\
\text { Neurociência de Decisão. }\end{array}$ & $\begin{array}{c}\text { University Southern California- } \\
\text { USC. }\end{array}$ \\
\hline André Palmini & $\begin{array}{l}\text { Epilepsia; Neurologia do Comportamento; Déficit de } \\
\text { Atenção; Hiperatividade; Doenças do Comportamento. }\end{array}$ & $\begin{array}{l}\text { Pontifícia Universidade Católica } \\
\text { do Rio Grande do Sul - PUCRS. }\end{array}$ \\
\hline $\begin{array}{l}\text { Augusto } \\
\text { Buchweitz }\end{array}$ & $\begin{array}{c}\text { Neurociências; Neuroeducação; Neurologia } \\
\text { Comportamental; Neuroética e Ciências Cognitivas. }\end{array}$ & $\begin{array}{l}\text { Pontifícia Universidade Católica } \\
\text { do Rio Grande do Sul - PUCRS. }\end{array}$ \\
\hline Álvaro Dias & $\begin{array}{l}\text { Cognição Social; Tomada de Decisão; Aplicação de } \\
\text { Técnicas de Neuroimagem em Psiquiatria. }\end{array}$ & $\begin{array}{l}\text { Universidade Federal de São } \\
\text { Paulo - USP }\end{array}$ \\
\hline
\end{tabular}

Fonte: Elaborado pelos autores (2015).

As entrevistas foram realizadas no nos anos de 2013 e 2014, sendo que o tempo de duração variou, em média, entre 30 minutos e 1 hora e 30 minutos. A fim de zelar pela ética na pesquisa qualitativa, e pela imagem pessoal e profissional dos especialistas, solicitou-se autorização para gravá-las e, posteriormente utilizar os dados na pesquisa. Nesse sentido, pontos das entrevistas que não foram autorizados pelos especialistas entrevistados foram cortados para fins de utilização de citações literais. Assim, os discursos aprovados foram transcritos para posterior análise dos dados (BARDIN, 2009).

Realizadas essas etapas, a análise dos dados compreendeu duas fases fundamentais: a análise de conteúdo e a triangulação de dados a partir da perspectiva teórica. Seguindo os pressupostos de Bardin (2009), a análise de conteúdo foi realizada da seguinte forma: (1) no primeiro momento, realizou-se a pré-análise dos dados mediante uma leitura flutuante; (2) em seguida, concretizou-se a codificação, categorização e/ou classificação dos dados com o suporte da revisão teoria; (3) por fim, consolidou-se o tratamento e a interpretação dos dados, destacando os pontos salientes que emergiram dos discursos dos especialistas entrevistados. Esse processo foi apoiado pelo software de análise de dados qualitativo MAXQDA. Baseando-se na concepção de Flick (2009), o processo de triangulação pode ser definido e, sobretudo, utilizado no contexto da pesquisa acadêmica para combinar métodos de pesquisa, grupos de estudo, ambientes locais e temporais e perspectivas teóricas distintas com o objetivo de investigar determinado fenômeno. Visto que o processo de triangulação dos dados 
não se limita a combinação de metodologias de pesquisa, nesse estudo foram combinadas diferentes perspectivas teóricas, advindas do campo do comportamento do consumidor e neurociências, além das percepções de especialistas que integram duas áreas de conhecimento: comunidade acadêmica de Marketing e Neurocientífica.

\section{A neurociência como método para compreender comportamentos de consumo}

No que tange a compreender o comportamento do consumidor a partir da ótica das abordagens neurocientíficas, o estudo de Solnais et al. (2013), por exemplo, traz uma importante revisão sobre "o que já foi feito", apresentando uma discussão interdisciplinar dos principais estudos e abordagens para a compreensão das esferas/dimensões que abrangem o estudo do comportamento do consumidor: tomada de decisão, processamento de recompensas, memória e emoções. Outras revistas científicas internacionais, não necessariamente da área de marketing, trouxeram contribuições sobre o tema nos anos 2000, como Journal of Economic Psychology (HUBERT, 2010), Neuroimage (DIMOKA, 2011), Brain Research Bulletin (WALTER et al., 2005) e Nature (ARIELY; BERNS, 2010).

O tipo de pesquisa hoje realizada em Comportamento do Consumidor geralmente passa pela interação com os sujeitos de pesquisa e quase que invariavelmente envolve a resposta (ou a reação) destes sujeitos a questionamentos ou estímulos. Esta resposta, em grande parte dos casos, envolve reflexões por parte dos sujeitos que se situam dentro dos limites da consciência. Damásio (2000, p. 18) defende que a consciência é o "problema supremo" para quem “deseja compreender os alicerces biológicos da mente”. Mas será suficiente? E os problemas fora dos limiares da consciência? Mlodinow (2012) afirma que só temos consciência de 5\% de nossa função cognitiva. Complementando essa ideia, Bechara e Damásio (2005) defendem que muitos acontecimentos são absorvidos apenas inconscientemente, sem o conhecimento consciente do sujeito. Neste sentido, diversos autores concordam que as tecnologias para pesquisa em neurociência, especialmente o fMRI (functional magnetic resonance imaging), contribuem para iluminar os processos inconscientes que guiam a tomada de decisões do consumidor, especialmente os aspectos afetivos (YOON et al., 2012; SHIV et al., 2005). A compreensão dos padrões de contingência que levam a emoções específicas é, segundo Foxall (2008), um pré-requisito essencial para as pesquisas que buscam determinar os mecanismos neurais responsáveis pelas emoções. Conforme destaca um dos especialistas:

Talvez, o grande objetivo do Neuromarketing seja descobrir um perfil correspondente a cada produto, que desencadeie um padrão de ativação 
cerebral [...], por exemplo, sempre que uma determinada música tocar, a pessoa vai dar um jeito de conseguir dinheiro para comprar aquilo, que nem um drogado, quando ele vê qualquer coisa que faça alusão à droga ele vai querer consumir [...] então, se o Neuromarketing descobrir um jeito de fazer uma campanha publicitária que forneça uma coisa recompensadora, de repente tu não vai resistir [...] eu desativo um pouco mais de uma determinada região cerebral e, com isso, a pessoa fica muito mais a mercê do estímulo [...] nesse jogo de ativação e desativação, tu pode fazer aquela combinação, que talvez module a decisão do indivíduo (Entrevistado 1).

Este método iria além das tradicionais mensurações de emoções por surveys que produzem categorias emocionais que não são funcionalmente relacionadas ao comportamento. Entretanto, autores como Payzan e Bourgeois-Gironde (2005) ponderam que a visualização das regiões neurais específicas que são ativadas durante experimentos controlados não permite per se que se correlacione a ativação da zona a um comportamento subsequente. Somente uma série de reaplicações e explanações sobre a mesma ocorrência poderia levar a associações entre uma determinada região neural e um padrão comportamental. Assim, os dados neuroeconômicos (os relativos a marketing também) seriam produto de um "processo de solidificação, e, se não solidificados, não poderiam pretender estabelecer resultados" (PAYZAN; BOURGEOIS-GIRONDE, 2005, p. 9).

A opinião de especialistas entrevistados reforça esses cuidados necessários para a compreensão e real contribuição que os métodos neurocientíficos aplicados ao Marketing podem trazer:

O que tem que ver é até que ponto vai avançar esse caminho da neurociência ligado ao marketing ao ponto de saberem que tipos de estímulos realmente abrem a combinação do cofre do indivíduo [...] a gente não sabe se vai acontecer, mas vai ter que ter junto um processo ético que ponha algum limite nessa questão. Eu não sou contrário a isso, mas é importante fazer essa reflexão (Entrevistado 2).

Outra questão bastante discutida refere-se ao realismo subjacente ao estudo, ou seja, a retirada do consumidor de seu ambiente natural de consumo, incluindo aí o seu elemento social. Este tipo de situação ocorre tanto em metodologias experimentais de causa e efeito, muito utilizadas nas áreas de comportamento de consumo, como nas abordagens neurocientíficas de coleta e análise de dados. Dois especialistas em marketing reforçam este ponto:

Um dos desafios do experimento é a questão do realismo, como é que eu 
trabalho o experimento de forma com que ele fique realista para o sujeito, como é que eu transformo um ambiente totalmente controlado que é para ter efeito de validade interna para um ambiente real ou mais próximo da realidade de consumo de tal forma que o sujeito não perceba os reais objetivos do estudo e que ele consiga atuar com naturalidade, como se estivesse atuando no seu dia-a-dia (Entrevistado 4).

Basicamente, quando a gente faz um experimento é uma caixa preta [...] você mostra alguns estímulos, olha a resposta e imagina um processo no meio do caminho, com base na construção teórica. [...] essa caixa preta também tem às suas limitações que podem ser vários processos (Entrevistado 5).

O discurso dos especialistas em marketing é consistente com os conceitos propostos por Engel, Kollat e Blackwell (1968) ao postularem que, sob o enfoque do método experimental de causa-efeito, os mecanismos subjacentes ao comportamento de decisão são considerados uma caixa preta, ou seja, inacessíveis à observação direta. Logo, reitera-se que uma das principais finalidades do método experimental consiste em analisar os dados observáveis que envolvem o processo de escolha, isto é, as respostas comportamentais cognitivas e afetivas - dos indivíduos frente a estímulos de marketing (VENKATRAMAN; PAYNE; 2011; FUGATE, 2007).

Assim, o uso de ferramentas da neurociência para predizer comportamentos de consumo poderia ajudar a abrir a chamada "caixa-preta" tão discutida pelos pesquisadores em comportamento do consumidor desde os primeiros modelos preditivos desenvolvidos nos anos 1960. A compreensão das áreas cerebrais que são estimuladas durante o processo de tomada de decisão permite que melhor se compreenda os achados dos chamados métodos acessados pelo consumidor, que guiam as ferramentas tradicionais de pesquisa em consumo. Os métodos que confiam principalmente na habilidade dos respondentes de "descrever e reconstruir sentimentos e pensamentos são muito subjetivos. Muitos efeitos no organismo humano que influenciam o comportamento não são percebidos conscientemente", segundo Hubert e Kenning (2008, p. 273). Neste sentido, a compreensão das necessidades emocionais inconscientes dos consumidores é o principal ganho dos estudos em neurociência do consumo.

Segundo Ariely e Berns (2010), pesquisas recentes indicam que os processos cognitivos associados às decisões de compra são multifatoriais e não podem ser reduzidos ou compreendidos por uma única área de ativação cerebral. Da mesma forma, uma dada região cerebral pode estar associada a uma série de processos cognitivos. Assim, as respostas dos consumidores aos esforços de marketing e as suas escolhas dependem de uma gama de processos neurobiológicos, e que uma única área cerebral não é responsável sozinha pelo 
processo de escolha do consumidor. A triangulação de métodos aparece aqui como uma alternativa que permite uma melhor compreensão dos processos de escolha. A carência da ligação entre os testes em ressonância magnética e as reais decisões de compra mensuradas posteriormente, ou outras medidas de consumo, faz com que este método sozinho ainda tenha muitas limitações. Plassmann et al. (2012) sugerem que a neurociência é uma ferramenta, dentre outras disponível para melhor compreender a psicologia por trás do comportamento de escolha. O uso da neurociência é especialmente útil nesta composição de ferramentas quando os processos investigados são difíceis de verbalizar ou manipular em situações como experimentos ou pesquisas survey.

Plassmann et al. (2012) salientam que o mais importante nas pesquisas futuras em neurociência de consumo é que a área vá além de simplesmente estabelecer associações entre uma dada região de ativação cerebral e um dado comportamento. Os autores citam um estudo de Kable (2011) que evidencia que entre 60 e $70 \%$ dos estudos empíricos que utilizaram neurociência para pesquisas comportamentais de tomada de decisão usaram apenas um método, a ressonância magnética funcional. Um dos principais problemas destacados por este método é a questão da "inferência reversa", amplamente discutida nos principais estudos da área (ARIELY; BERNS, 2010; DIMOKA, 2011), que ocorre quando o "engajamento em um processo mental particular é inferido pela ativação de uma região cerebral em particular" (PLASSMANN et al., 2012, p. 29 referenciando POLDRACK, 2006). Os autores salientam que a validade dedutiva de tais inferências é limitada. Discutir o processo de inferência reversa não é objetivo deste trabalho, porém uma importante consideração é o fato de que uma única área cerebral pode ser multitarefa, e neste caso o cérebro produz uma redundância, não sendo possível aferir a que tarefa um dado processamento está se referindo. Portanto, se uma determinada área cerebral é responsável por uma série de processos mentais, a ativação daquela área é uma evidência fraca da ativação de um dado processo. O contrário ocorre se uma dada área é associada a um processo mental específico.

Um esforço no sentido de unificar técnicas e suplantar as limitações da inferência reversa é dado por Dimoka (2011). No estudo da autora, escalas psicométricas já validadas na literatura para a mensuração de processos psicológicos em ciências sociais são utilizadas ao mesmo tempo que a ressonância magnética funcional. Ao passo que os sujeitos são solicitados a avaliar um grau de dado processo psicológico na escala, a ressonância mensura a área cerebral ativada por este processo psicológico focal. Assim, a autora buscava validar as correlações neurais dos processos psicológicos que já tinham sido identificados na literatura, 
permitindo assim a comparação entre as correlações entre ativações neurais objetivas e suas medidas subjetivas correspondentes. A convergência metodológica entre diferentes técnicas neurocientíficas e "tradicionais" de pesquisa de mercado, assim como a reaplicação e validação dos resultados parecem indicar os caminhos futuros da pesquisa na área (HUBERT, 2010), levando à expansão da caixa de ferramentas neurocientíficas (PLASSMANN et al., 2012).

Payzan e Bourgeois-Gironde (2005) salientam que a chave para melhor compreender a "caixa-preta" do comportamento é a unificação das ciências comportamentais, envolvendo o sincronismo entre neurologistas, psicólogos, filósofos e outros pesquisadores. A colaboração entre neurocientistas, cientistas cognitivos e cientistas sociais seria benéfica a todos os interessados no estudo do comportamento, trazendo explanações mais compreensíveis sobre o comportamento e a mente humanas, segundo Cacioppo (2002). Lee, Broderick e Chamberlain (2007) também clamam por uma maior colaboração entre departamentos e escolas nos estudos da área. Senior et al. (2007) destacam, ainda, a importância do "pluralismo metodológico" dos pesquisadores da área.

Tal desafio fica evidente na fala de um dos entrevistados, reforçando a difícil tarefa de convergir esforços de diferentes áreas do conhecimento:

Quem detém essa tecnologia e esse conhecimento, os neurocientistas e os psicólogos cognitivos têm outras preocupações, fazer o cara viver mais, a cura do câncer, que poderiam trazer algum ganho e explicação aos nossos fenômenos, às vezes tem uma visão deturpada de Marketing [...] que é venda, só propaganda, que só quer empurrar as coisas [...] Talvez um bom começo seria fazer parcerias com algum alinhamento de pesquisa, mas para isso os neurocientistas também devem compreender que Marketing e consumo também são fenômenos importantes [...] Um caminho seria olhar para os fenômenos de compra que são relevantes para esses pares, por exemplo, a ideia do comportamento de compra compulsivo (Entrevistado 4).

\section{Questões éticas na neurociência aplicada ao consumo}

É notório o crescimento das preocupações acerca da ética na pesquisa, em um sentido amplo, bem como em torno dos estudos sobre os comportamentos de consumo, em particular. Contudo, a convergência das neurociências com a pesquisa em marketing determina um nível de atenção aos aspectos éticos significativamente acima dos atualmente praticados, levando a necessidade de um código de ética específico para atender aos desafios da neurociência aplicada ao consumo (MURPHY; ILLES; REINER, 2008). Assumindo-se que esta linha de pesquisas depende, primordialmente, de uma colaboração próxima entre pesquisadores de 
marketing e neurocientistas, as barreiras determinadas por diferentes concepções éticas tornam-se ainda mais relevantes (LEE et al., 2007).

Adicionalmente, a falta de consenso sobre a aceitação do uso da neurociência como parte dos esforços de pesquisa em marketing - em especial entre neurocientistas - impõe a necessidade de uma discussão ampla, estabelecendo limites que evitem a percepção de abusos que levem à suposta capacidade de "ler as mentes dos consumidores" e de "manipular seus comportamentos" (HUBERT; KENNING, 2008). Perrachione e Perrachione (2008) destacam que o ceticismo por parte de alguns neurocientistas se deve em parte ao papel inerente aos cientistas, que devem revisar criticamente novas propostas de pesquisa; entretanto, outra parte seria uma falha dos pesquisadores em consumo em comunicar o que exatamente a área busca, sob o ponto de vista de pesquisa.

No decorrer das entrevistas realizadas como parte deste trabalho, evidenciou-se a preocupação dos entrevistados com os aspectos éticos relativos à pesquisa:

Tem que ver quais são os limites éticos desse tipo de pesquisa, porque isso ainda está muito aberto, tanto que algumas instituições ficam horrorizadas com essa ideia de Neuromarketing e outras estão capitalizando muito em cima disso [...] Toda publicidade é um pouco de manipulação, a grande questão é o que é mais e o que é menos aceitável, por exemplo, os jogo em cassino [...] se tu tem um cassino que faz uma propaganda com gente bonita, um ambiente legal, tu está manipulando as pessoas irem lá e jogarem. Eu não posso estimular as pessoas a jogarem porque a lei Brasileira proíbe. Eu sou contrário a isso, sem que haja uma discussão ética subjacente, no qual as pessoas fiquem protegidas de um tipo de estimulação que elas não tenham como resistir (Entrevistado 1).

Por outro lado, discussões trazidas por Murphy, Illes e Reiner, (2008) e Ariely e Berns (2010) apontam para a cautela no que se refere às reais ameaças decorrentes do uso de técnicas neurocientíficas em marketing, na medida em que seus resultados são, em geral, superestimados por seus críticos. Farah et al. (2008), abordando implicações do uso de neuroimagens na psicologia, concluem que precauções sobre confidencialidade das informações devem ser tomadas, mas questionam até que ponto existem, por exemplo, diferenças entre a "privacidade comportamental" e a "privacidade do cérebro" e ressaltam o equilíbrio necessário entre privacidade individual e benefícios à sociedade. Por certo, pesquisadores na área de marketing que venham a investir no uso de técnicas de neurociências para melhor entendimento dos comportamentos de consumo terão de rever diferentes parâmetros de condução de suas pesquisas. 
Illes et al. (2010), em um estudo exploratório junto a pesquisadores da área de neurociências, agruparam questões éticas em quatro fatores: confidencialidade/consentimento (incluindo privacidade), fatores externos de produtividade acadêmica (prioridades de pesquisa, opinião pública e fontes de financiamento), conflito de interesse (conflitos entre interesses acadêmicos e comerciais) e vulnerabilidade/expectativas (expectativas excessivas quanto aos resultados, uso de participantes integrantes de grupos vulneráveis e achados clínicos inesperados). Garnett et al. (2011) apontam que questões éticas em estudos com uso de fMRI emergem desde a concepção do experimento até a interpretação e aplicação de seus resultados. Consequentemente, a atenção à ética é requerida em todos os estágios de desenvolvimento de uma pesquisa, sendo possível dividir diferentes considerações éticas em três momentos: planejamento da pesquisa, desenvolvimento do estudo, divulgação e aplicação dos resultados.

Em relação ao planejamento da pesquisa, em que pese a Resolução no 196/96 do Conselho Nacional de Saúde/Ministério da Saúde (CNS/MS) determinar que "toda pesquisa envolvendo seres humanos deverá ser submetida à apreciação de um Comitê de Ética em Pesquisa", no campo específico das pesquisas em comportamento do consumidor as exigências relativas à análise prévia de projetos de pesquisa são bastante superficiais, geralmente limitando-se a cuidados com o sigilo das informações e à apresentação do Termo de Consentimento Livre e Esclarecido assinado pelos participantes.

Considerando ser natural a interface entre academia e organizações no contexto dos estudos de marketing, um componente preliminar fundamental envolve a declaração de conflito de interesse (FISCHER et al. 2010), o qual ocorre em situações em que pesquisadores possuem interesses que não estão completamente explícitos, mas podem influenciar em sua conduta em uma pesquisa. Tais conflitos podem ser de natureza pessoal, comercial, política, acadêmica ou financeira e são destacados no Manual de Ética Médica da World Medical Association (WMA, 2009). Outro aspecto importante refere-se às técnicas de mapeamento que o pesquisador pretende adotar. Ainda que as técnicas empregadas sejam em geral não invasivas e de baixo risco, os procedimentos de um estudo que utiliza EEG (eletroencefalografia) são distintos quando comparados ao uso de fMRI e impactam os participantes.

Você tem que recrutar pessoas que tenham interesse em passar por um procedimento dentro de um hospital, depois essas pessoas têm que ser saudáveis, que não tenham nenhum problema com depressão, que não tomam 
nenhum medicamento, que não tenham tatuagem porque não pode entrar na máquina, que não tenham nenhum metal no corpo [...] você tem que deixar a pessoa se acalmar dentro da máquina porque é um ambiente completamente diferente de um ambiente de loja. A pessoa acaba entrando no clima, mas no início é difícil. A pessoa tem medo, ela pode ser claustrofóbica, ela está emocionalmente diferente, ela não vai estar com a roupa dela, ela vai ter que vestir um avental de um hospital (Entrevistado 5).

Torna-se, assim, necessário um completo detalhamento prévio de objetivos, envolvimento requerido dos participantes, estímulos a serem utilizados, riscos eventualmente existentes aos participantes, bem como de uma descrição precisa do perfil da amostra a ser pesquisada. O projeto, ou protocolo de pesquisa, só então será passível de submissão a um Comitê de Ética na Pesquisa.

A descrição quase anedótica feita por Morin (2011) relatando os passos e sensações de um hipotético participante de um estudo submetido à fMRI ressaltam a natureza absolutamente distinta da pesquisa baseada em técnicas de neurociências em relação às tradicionais técnicas de pesquisa em marketing. Assim, cuidados adicionais devem ser compreendidos e assumidos ao longo do processo de desenvolvimento do estudo. Particularmente, as preocupações centrais recaem na proteção física e mental dos participantes frente a riscos inerentes ao estudo (MURPHY; ILLES; REINER, 2008) e à preservação da privacidade do indivíduo (FARAH et al. 2008). Por exemplo, a permanência em um equipamento de fMRI durante 30 minutos pode gerar razoáveis níveis de estresse em certas pessoas, o que determina cuidados adicionais na seleção, informação prévia e condução do estudo.

As questões ligadas à privacidade dos participantes são um tanto mais complexas, pois envolvem "potenciais ameaças à privacidade do cérebro pelo uso de neuroimagens funcionais" (FARAH et al. 2008, p. 119). O dilema ético aqui remete à forma e ao estado de consciência do indivíduo no momento em que são coletados os dados. Em um exame de fMRI os dados são coletados em uma situação na qual o participante não tem o conhecimento nem a consciência plena do que de fato está sendo monitorado, fazendo com que o pesquisador “invada" domínios desconhecidos pelo próprio indivíduo. Além disto, há o risco inerente de o estudo chegar a resultados inesperados do ponto de vista clínico, apontando desordens ou doenças não detectadas previamente, pois no momento de um mapeamento de imagens, diferentes áreas do cérebro estarão sendo monitoradas.

Ainda no escopo dos aspectos éticos durante o desenvolvimento dos estudos, a seleção adequada de participantes representa um elemento destacado na literatura (LEE et al., 2007; 
MURPHY; ILLES; REINER, 2008; ARIELY; BERNS, 2010), pois considerável preocupação repousa sobre a participação de indivíduos pertencentes a grupos vulneráveis, tais como crianças ou pessoas portadoras de alguma desordem cognitiva ou comportamental. Tal preocupação igualmente se manifesta na ótica dos entrevistados:

Essa coisa de tu ter uma ideia do que está passando na cabeça de uma pessoa é uma coisa um pouco delicada porque todas as ações humanas podem ser usadas para o bem e para o mal. Para o bem, funciona assim: vamos imaginar que tu queiras fazer uma campanha para que as pessoas doem órgãos, então se tu tiveres uma noção do melhor jeito de fazer uma campanha que "pegue" ou "capte" o maior número possível de pessoas seria o ideal. Por outro lado, tu tens que ter um pouco de cuidado, porque a maioria das pessoas na nossa sociedade tem um nível educacional muito baixo e são mais facilmente manipuláveis, daqui a pouco tu estás cheio de gente se afundando em dívidas porque tu consegues mapear como elas se sentem mais atraídas (Entrevistado $1)$.

Considerando o nível de esclarecimento e compreensão prévia dos propósitos do estudo (comentado anteriormente), o contexto brasileiro apresenta exemplos nítidos de risco de exposição de grupos vulneráveis, tais como consumidores de baixa renda e/ou grau de instrução e que têm sido alvo prioritário dos estudos na área em anos recentes. Murphy, Illes e Reiner, (2008) destacam ainda o problema das recompensas financeiras para participantes de estudos de marketing, o que pode induzir o indivíduo a uma análise distorcida, mascarando eventuais riscos à participação no estudo.

Dentre as três etapas de uma pesquisa aqui analisadas, a que mais suscita críticas e debates acerca da ética nos estudos de neurociências e marketing refere-se ao uso e divulgação dos resultados. Enquanto o debate ético na pesquisa nas etapas de planejamento e desenvolvimento do estudo permeia toda a área médica, em particular os neurocientistas, os questionamentos sobre as aplicações dos resultados recaem mais fortemente em áreas que se utilizam das técnicas neurocientíficas com propósitos outros que não o da compreensão e/ou cura de patologias humanas. E neste contexto, o marketing torna-se o alvo preferido.

Manifestações críticas acerca do uso de informações obtidas através da abordagem neurocientífica com fins manipuladores são citadas com frequência na literatura como o principal foco dos críticos à neurociência do consumo, incluindo a busca pelo chamado "buy bottom" (MORIN, 2011), a criação de campanhas publicitárias absolutamente irresistíveis (LEE et al., 2007), a manipulação dos comportamentos de consumo (MURPHY; ILLES; REINER, 2008) e a “criação de legiões de zumbis consumidores controlados por corporações 
onipotentes que usam a neuroimagem para criar propagandas hiperefetivas" (SENIOR; LEE, 2008, p. 263).

Murphy, Illes e Reiner, (2008) chamam de stealth neuromarketing o potencial risco do uso de informações obtidas por métodos da neurociência para manipular comportamentos humanos. Central nesta discussão está o conceito de autonomia, ou seja, até que ponto as "ferramentas de neuromarketing fornecerão elementos suficientes relacionados às funções neurais humanas de modo a permitir a manipulação do cérebro de tal forma que o consumidor será incapaz de detectar tais subterfúgios" (p. 297), determinando que ao menos alguns grupos de indivíduos tenham exatamente os comportamentos desejados pelas organizações.

Walter et al. (2005), Lee et al. (2007), Murphy, Illes e Reiner (2008), Fisher et al. (2010) e Ariely e Berns (2010) destacam que os atuais recursos neurocientíficos não permitem que se atinja tal ponto de compreensão e, principalmente, manipulação do comportamento humano. Contudo, torna-se imprescindível que estudiosos e profissionais de marketing atentem para tais questões ao divulgar e aplicar seus resultados. Esta questão encontra paralelo também nas limitações de estudos neurocientíficos quanto à validade e à generalização de seus resultados.

Uma revisão de estudos sobre neurociência do consumidor, feita por Hubert e Kenning (2008), apresenta estudos que utilizaram amostras que variaram entre 3 e 67 participantes, com uma maioria de estudos com $\mathrm{n}<30$. Para Kenning e Linzmajer (2011), uma importante limitação (e suas decorrentes consequências) ao uso da neurociência no estudo do consumo é o alto custo financeiro e de tempo para a realização de pesquisas. Isto implica, geralmente, amostras pequenas, dificultando a generalização dos resultados obtidos e aumentando a possibilidade de ocorrência de falsos positivos. Embora as questões de validade externa e generalização de resultados sejam similares aos tradicionais estudos experimentais já realizados em comportamento do consumidor, a complexidade do funcionamento do cérebro e a amplitude de uso dos resultados de estudos a ele associados ainda requerem extremo cuidado por parte de pesquisadores no que tange à extrapolação dos achados, ressaltando a importância das realizações (HUBERT, 2010).

Outro aspecto relevante em termos de contribuições resultantes de estudos com abordagem neurocientífica está na relação custo-benefício de tais pesquisas. Conforme aponta um dos entrevistados:

Agora o que eu acho também, quer dizer, eu tenho certeza, é que quando você fala em Neuromarketing tem muita coisa errada sendo feita [...] tem muita 
coisa que eles estão dizendo com medidas cerebrais que poderiam ser ditas com medidas subjetivas com um custo bem mais baixo [...] então, para se fazer pesquisa aplicada às ciências sociais e essencialmente no comportamento do consumidor, você essencialmente precisa de bons neurocientistas, alguém que entende muito dessa área (Entrevistado 3).

A eventual excessiva ênfase ao uso das neurociências também é mencionada, sob outro olhar:

Eu acho que o que ocorre muito hoje em dia é a ideia de: "ahhh isso é uma coisa nova, vai ser legal", então os revisores vão gostar porque é novidade, mas tem que pensar que tipo de contribuição esse método te permite fazer que tu não poderias fazer usando um método mais simples e mais barato (Entrevistado 6).

O que acontece hoje em dia no Brasil é que tem uma onda enorme de gente que fica falando de Neuromarketing e blá blá blá, mas no fundo, no fundo, o que faz na prática? Vende palestras, entende? Vai lá para falar de Neuromarketing, fingir que faz, que usa as ferramentas, que está vendendo no mercado, eu acho isso tudo completamente errado do ponto de vista ético (Entrevistado 3).

As questões acima levantam um importante questionamento acerca da real necessidade de serem empregadas técnicas neurocientíficas em estudos onde técnicas tradicionais de pesquisa em marketing podem ser suficientes, apontando também para uma noção de "modismo" das neurociências como enfoque para estudo do comportamento do consumidor.

Vindo na contramão das críticas à neurociência do consumo, conforme Achrol e Kotler (2012) destacam, o marketing não diz mais respeito a consumidores individualmente, mas a sociedade como um todo. Assim, o aprofundamento dos conhecimentos advindos da interface entre neurociências e marketing abre uma importante trilha de oportunidades para a compreensão de comportamentos desviantes, tais como consumo compulsivo, compra por impulso, aspectos de ansiedade e autoestima associados ao consumo, educação para o consumo e consumo consciente. Tais temas somente poderão ser endereçados com propriedade pelos profissionais de marketing, na medida em que sejam compreendidos os processos internos que ocorrem no cérebro e determinam tais comportamentos. Este assunto é discutido no tópico a seguir.

\section{Possibilidades de pesquisa e interesses para a sociedade}

Apesar da percepção por alguns grupos da natureza "má" do marketing, o objetivo principal, desde os primórdios da disciplina, é conciliar produtos mais adequados às 
preferências do consumidor e facilitar o processo de decisão do mesmo (ARIELY; BERNS, 2010). Os autores destacam que ao mesmo tempo que há temores sobre o uso da neurociência para descobrir alimentos tão saborosos que não seria possível resistir a eles, também há a possibilidade de que as mesmas técnicas de pesquisa sejam utilizadas para trazer maior interesse a alimentos nutritivos. Novamente o uso e não a técnica é que parece estar em discussão.

Um possível caminho a ser trilhado é buscar a convergência de interesses de pesquisadores das diferentes áreas. Neste sentido, que tipo de fenômenos poderia atrair pesquisadores em comportamento do consumidor e neurociência? Em que contextos pode haver interseção de problemas de pesquisa, realmente relevantes a ambos? Ou que envolvam ainda outros pesquisadores, como psicólogos, sociólogos ou antropólogos? Algumas possibilidades, às vezes referidas como "o lado negro do consumo", estão relacionadas aos comportamentos aberrantes do consumidor (BUDDEN; GRIFFIN, 1996). Tais comportamentos, segundo Fullerton e Punj (1993, p. 570), referem-se a "comportamentos em situações de troca que violam normas de conduta geralmente aceitas nestas situações”. Entre as possibilidades de estudo, sugere-se:

1) furtos em lojas: o furto executado pelo consumidor é considerado um dos comportamentos mais aberrantes, trazendo impactos negativos para as empresas e para a sociedade. De acordo com Babin e Babin (1996), além dos motivadores cognitivos e éticos para o furto, as emoções ocupam espaço importante como preditoras deste tipo de comportamento. Quais tipos de emoções são mais relevantes e como elas desencadeiam o furto?

2) compra compulsiva: reflete um comportamento que foge do controle do consumidor, gerando uma vontade irresistível de comprar (ELLIOTT; ECCLES; GOURNAY, 1996). Segundo O’Guinn e Faber (1989, p. 155), compras compulsivas são “compras crônicas e repetitivas que se tornam respostas primárias a eventos ou sentimentos negativos". Quais são os motivos que levam à compra compulsiva (HASSAY; SMITH, 1996)? Como lidar com os sentimentos de culpa, ansiedade e vergonha decorrentes desse tipo de comportamento (McELROY et al. 1994)?

3) compra impulsiva: Rook e Fischer (1995) indicam que mais de $25 \%$ dos consumidores são impulsivos, ou seja, tendem a realizar compras não planejadas, não intencionais, imediatas e sem reflexão (SANTINI, 2013; WEUN; JONES; BETTY, 1998) que, em altos níveis, podem ser potencialmente autodestrutivas (SILVERA; LAVACK; 
KROPP, 2008). Relacionando comportamentos impulsivos com a saúde do consumidor, pode-se verificar a relação da compra impulsiva com o estresse ou com déficit de atenção (KAUFMAN-SCARBOROUGH; COHEN, 2004). Além disso, existem indicativos que características específicas de alguns alimentos guardam relação com compras por impulso (MISHRA; MISHRA, 2010).

4) vícios do consumidor: a compra compulsiva, discutida acima, também é considerada um tipo de vício (na experiência de consumo em si). Estendendo as possibilidades, podem ser focos de pesquisa os vícios em apostas, em pornografia, em álcool e em drogas, e seus impactos na qualidade de vida do consumidor (ELLIOTT; ECCLES; GOURNAY, 1996).

5) distúrbios alimentares: alguns consumidores respondem a estados emocionais (raiva ou tristeza, por exemplo) comendo de forma desenfreada (KEMP; BUI; GRIER, 2011). Quais são os mecanismos neurais ativados pelos emotional eaters? Comportamentos alimentares pouco saudáveis, comuns em nossa sociedade, como alimentos gordurosos, quantidades excessivas de alimento ou realizar refeições sistematicamente fora de casa podem levar à obesidade. A obesidade epidêmica é decorrência de uma falha mercadológica (CHARLEBOIS; TAMILIA; LABRECQUE, 2007)? Qual o impacto do food marketing (SEIDERS; PETTY, 2004)? Mais especificamente, qual o impacto das propagandas de alimentos na obesidade (EAGLE et al., 2004; GARDE, 2008)?

6) endividamento do consumidor: o endividamento de domicílios e consumidores é uma preocupação social e foco de pesquisa de muitos pesquisadores (TURINETTI; ZHUANG, 2011). Ottaviani e Vandone (2011) identificaram relação entre o endividamento e a impulsividade do consumidor. Os cartões de crédito, por exemplo, ao mesmo tempo que representam uma tecnologia que facilita as transações comerciais, trazem consigo riscos de compras compulsivas ou impulsivas e de acumulação excessiva de dívidas por parte do consumidor (BERNTHAL; CROCKETT; ROSE, 2005).

7) comprar como terapia: alguns consumidores usam o ato de comprar como forma de melhorar o humor ou "passar o tempo" (ATALAY; MELOY, 2011). Quais as possíveis consequências desse comportamento?

Vale destacar que o entendimento dos itens acima propostos tem relevância em vários sentidos, além dos interesses dos pesquisadores em neurociência e comportamento do consumidor. Existem consequências nítidas ao estabelecimento de políticas públicas, na área 
da saúde e na proteção do consumidor, além de um propósito mais amplo, qual seja o de proporcionar maior qualidade de vida aos sujeitos.

\section{Considerações e implicações}

As maiores contribuições das neurociências ao entendimento dos comportamentos de consumo certamente ainda estão por vir; contudo, para que se assegure que tais contribuições sejam positivas e benéficas não apenas para grupos com interesses restritos, inúmeras decisões e ações devem ser adotadas desde já.

O presente artigo buscou aprofundar questões centrais que norteiam as discussões acerca do uso de técnicas neurocientíficas no estudo do comportamento do consumidor em convergência com uma série de entrevistas de profundidade junto a especialistas. Uma primeira consideração final relevante é o alinhamento da visão dos especialistas com os pontos-chave destacados na literatura, sobretudo no que tange a limitações/cuidados metodológicos e fronteiras éticas neste tipo de pesquisa. Tendo em vista os aspectos destacados ao longo do texto, a seguir apresenta-se um detalhamento dos principais achados.

Um primeiro elemento importante é a adequada convergência entre neurociências e marketing. Para Antonietti e Iannello (2011), a simples justaposição de duas visões de um mesmo problema ou fenômeno não traz progresso à ciência (por exemplo, a psicologia do consumo explicando os processos mentais e a neurociência explicando tais processos de acordo com o funcionamento do cérebro). Entretanto, Damásio (2000, p. 30) afirma que:

No decorrer das duas últimas décadas, o trabalho em neurociência cognitiva tornou-se especialmente frutífero, pois o desenvolvimento de novas técnicas para observar o cérebro, visando conhecer sua estrutura e função, permite-nos agora associar determinado comportamento que observamos, clinicamente ou em um experimento, não só a um correlato mental presumido desse comportamento, mas também a marcadores específicos de estrutura ou atividade cerebral.

Entende-se que, considerando as possibilidades de integração expostas ao longo deste artigo, tal justaposição representa um desafio inicial para a reflexão sobre futuros avanços na pesquisa científica nas áreas de marketing e de consumo. Segundo Perrachione e Perrachione (2008, p. 315), a despeito do impacto das imagens cerebrais que os estudos em neurociência provocam, a primeira questão que os pesquisadores em marketing devem responder é: "nós realmente precisamos de imagens cerebrais para responder a nossa questão? Ou podemos trazer uma resposta comportamental ao trabalho de maneira mais rápida, acurada e menos 
cara?" Walter et al. (2005, p. 378) destacam o cuidado de não abusar da autoridade da neurociência em nossa sociedade: "isto não significa apenas evitar o ferimento. Isto também significa que nós devemos estar cientes das nossas responsabilidades de não abusar da neurociência induzindo medos inapropriados e esperanças que nunca serão preenchidas." Nas entrevistas realizadas com especialistas evidenciou-se também que as técnicas neurocientíficas não podem ser assumidas como o "santo graal" dos estudos de consumo. Antes de serem consideradas, os pesquisadores da área devem se questionar o quanto tais técnicas são necessárias e, também, o quanto podem de fato agregar aos conhecimentos já existentes e obtidos por técnicas mais tradicionais. Ainda, devem questionar se os padrões obtidos através de técnicas neurocientíficas são efetivamente sólidos e capazes de reproduzir o comportamento humanos em diferentes contextos de decisões de consumo, dadas suas limitações de artificialismo e validade externa.

O desenvolvimento da neurociência do consumo passa necessariamente por um alinhamento entre a consistência e a relevância de seus modelos de pesquisa com padrões éticos de elevado nível. Neste sentido, universidades, governo e entidades representativas têm a importante missão de incluir em suas agendas a discussão e o desenvolvimento de manuais ou códigos de conduta ética na pesquisa especificamente voltados a esta área. Em que pese a existência de códigos de ética na pesquisa, em geral tais orientações são genéricas e até o momento não contemplam as especificidades geradas pela interface entre a neurociência e o marketing (MURPHY; ILLES; REINER, 2008; FISCHER et al., 2010).

Igualmente a formação de pesquisadores na área passará por transformações. Achrol e Kotler (2012) destacam que os estudiosos de marketing e do consumidor necessitarão de conhecimentos relacionados à neurofisiologia como base para o desenvolvimento de seus estudos, bem como de uma postura mais ampla quanto aos desafios e contribuições do marketing na sociedade. Neste sentido, a formação de pesquisadores e profissionais de marketing deverá contemplar as bases para uma conduta ética no desenvolvimento de pesquisa e na aplicação de seus resultados. Cada vez mais a qualidade de um trabalho não será apenas medida pela competência técnica de seu desenvolvimento, mas também pela competência ética.

A construção de teorias a partir de estudos de base neurológica, embora não necessariamente utilizando técnicas neurocientíficas, é um dos futuros da união entre neurociência e marketing (ARIELY; BERNS, 2010). Da mesma forma, a tradução de constructos teóricos em categorias neurofisiológicas é igualmente desafiadora. Entretanto, é 
importante aceitar que este é um processo que leva tempo e não será uma revolução, mas um processo evolucionário que permitirá o casamento entre ciências e técnicas de pesquisa (HUBERT, 2010). Por certo, é possível afirmar que a empolgação inicial determinada pelos avanços tecnológicos e suas aplicações ao estudo do consumo passa por um momento de reflexão e de realinhamento de expectativas frente não apenas aos desafios enfrentados, mas também às limitações já observadas no campo empírico.

$\mathrm{Na}$ esfera estritamente gerencial, encoraja-se que as empresas de pesquisa de mercado que utilizam técnicas neurocientíficas deem ciência das suas pesquisas, ao menos dos descritivos metodológicos, de forma a permitir a avaliação da qualidade e da eficácia dos estudos por parte da comunidade científica. Para Ariely e Berns (2010), as contribuições da neurociência devem ser debatidas e discutidas pela comunidade acadêmica, caso contrário corre-se o risco de as "empresas de neuromarketing" tornarem-se rapidamente assunto do passado. Como os métodos utilizando neurociência aplicada são complexos, a sua utilização pelas empresas pode levar a dados inacurados, más interpretações e desapontamentos (HUBERT, 2010). Assim, a missão da comunidade científica seria permitir esta transição ou tradução dos resultados obtidos para as organizações. Ou seja, permitir que a neurociência de consumo se transforme efetivamente em práticas de marketing. 


\section{REFERÊNCIAS}

ACHROL, Ravi; KOTLER, Philip. Frontiers of the Marketing Paradigm in the Third Millennium. Journal of the Academy of Marketing Science, v. 40, p. 35-52, 2012.

ANTONIETTI, Alessandro; IANNELLO, Paola. Social science and Neuroscience: a Circular Integration. International Review of Economy, v. 58, p. 307-317, 2011.

ARIELY, Dan; BERNS, Gregory S. Neuromarketing: the Hope and Hype of Neuroimaging in Business. Nature Reviews Neuroscience, v. 11 (4), p. 284-292, 2010.

ATALAY, A. Selin; MELOY, Margaret G. Retail Therapy: a Strategic Effort to Improve Mood. Psychology \& Marketing, v. 28 (6), p. 638-660, 2011.

BABIN, Barry J.; BABIN, Laurie A. Effects of Moral Cognitions and Consumer Emotions on Shoplifting Intentions. Psychology \& Marketing, vol. 13 (8), 785-802, 1996.

BARDIN, L. Análise de conteúdo. Lisboa, Portugal: Edições 70, 2009.

BECHARA, A.; DAMASIO, A. R. The Somatic Marker Hypothesis: a Neural Theory of Economic Decision. Games and Economic Behavior, v. 52, p. 336-372, 2005.

BERNTHAL, Matthew; CROCKETT, David; ROSE, Randall L. Credit Cards as Lifestyles Facilitators. Journal of Consumer Research, v. 32, p. 130-145, Jun. 2005.

BOURGEOIS-GIRONDE, Sacha. Is Neuroeconomics Doomed by the Reverse Inference Fallacy? Mind Society, v.9, p. 229-249, 2010,

BUDDEN, Michael C.; GRIFFIN, Thomas F. Explorations and Implications of Aberrant Consumer Behavior. Psychology \& Marketing, vol. 13 (8), p. 739-740, 1996.

CACIOPPO, John, T. Social Neuroscience: Understanding the Pieces Fosters Understanding the Whole and Vice-Versa. American Psychologist, v, 57 (11), p.820-831, 2002.

CHARLEBOIS, Sylvain; TAMILIA, Robert D.; LABRECQUE, JoAnne. Food Marketing and Obesity: a Public Policy and Channels Perspective. Innovative Marketing, v. 3 (1), p. 83-99, 2007.

CROMBY, John. Integrating Social Science with Neuroscience: Potential and Problems. BioSocieties, v. 2, pp. 149-169, 2007.

DAMÁSIO, António. O mistério da consciência. São Paulo: Companhia das Letras, 2000. 
DIMOKA, Angelika. Brain Mapping of Psychological Processes with Psychometric Scales: an Fmri Method for Social Neuroscience. Neuroimage, v. 54, p. 263-271, 2011.

EAGLE, Lynne; BULMER, Sandy; KITCHEN, Philip; HAWKINS, Jacinta. Complex and Controversial Causes for the Obesity Epidemic: The Role of Marketing Communications. International Journal of Medical Marketing, v. 4 (3), p. 271-287, 2004.

ELLIOTT, Richard; ECCLES, Sue; GOURNAY, Kevin. Revenge, Existential Choice, and Addictive Consumption. Psychology \& Marketing, v. 13 (8), 753-768, 1996.

ENGEL, J.; KOLLAT, D.; BLACKWELL, R. Consumer Behavior. Holt, Rinehart and Winston, Inc. Dec, 1968.

FARAH, Martha; SMITH, Elizabeth; GAWUGA, Cyrena; LINDSELL, Dennis; FOSTER, Dean. Brain Imaging and Brain Privacy: a Realistic Concern? Journal of Cognitive Neuroscience, v. 21 (1). p. 119-127, 2008.

FISCHER, Carl; CHIN, Lisa; KLITZMAN, Robert. Defining Neuromarketing: Practices and Professional Challenges. Harvard Review of Psychiatry, v. 18 (4). p. 230-237, 2010.

FLICK, U. Introdução à pesquisa qualitativa. 3 ed. Porto Alegre: Bookman, 2009.

FOXXAL, Gordon R. Reward, Emotion and Consumer Choice: from Neuroeconomics to Neurophilosophy. Journal of Consumer Behaviour, v. 7 (4/5), p. 368-396, July-October 2008.

FUGATE, Douglas L. Neuromarketing: a Layman's Look at Neuroscience and its Potential Application to Marketing Practice. Journal of Consumer Marketing, v. 24 (7), p. 385-394, 2007.

FULlERTON, R. A., PUNJ, G. Choosing to Misbehave: a Structural Model of Aberrant Consumer Behavior. In: L. McAlister \& M. L. Rothschild (Eds.), Advances in Consumer Research, v. 20, p. 570-574. Provo, UT: Association for Consumer Research, 1993.

GARCIA, Justin R.; SAAD, Gad. Evolutionary Neuromarketing: Darwinizing the Neuroimagen Paradigm for Consumer Behavior. Journal of Consumer Behaviour, v. 7, p. 397-414, 2008.

GARDE, Amandine. Food Advertising and Obesity Prevention: what Role for the European Union? Journal of Consumer Policy, v. 31(1), p. 25-44, 2008.

GARNETT, Alex; WHITELEY, Louise; PIWOWAR, Heather; RASMUSSEN, Edie; ILLES, Judy. Neuroethics and fMRI: Mapping a Fledgling Relationship. PloS ONE, v. 6, (4), April, 2010 . 
HASSAY, Derek N.; SMITH, Malcolm C. Compulsive Buying: an Examination of the Consumption Motive. Psychology \& Marketing, v. 13 (8), p. 741-752, 1996.

HUBERT, Mirja. Does Neuroeconomics Give New Impetus to Economic and Consumer Research? Journal of Economic Psychology, v. 31, p. 812-817, 2010.

HUBERT, Mirja; KENNING, Peter. A Current Overview of Consumer Neuroscience. Journal of Consumer Behaviour, v. 7, p. 272-292, 2008.

ILLES, Judy; TAIRYAN, Kate; FEDERICO, Carole; TABET, Aline; GLOVER, Gary. Reducing Barriers to Ethics in Neuroscience. Frontiers in Human Neuroscience, v. 4, p. $167,2010$.

KABLE, Joseph W. The Cognitive Neuroscience Toolkit for the Neuroeconomist: a Functional Overview. Journal of Neuroscience, Psychology, and Economics, v. 4 (2), p. 63-84, 2011.

KAUFMAN-SCARBOROUGH, Carol; COHEN, Judy. Unfolding Consumption Impulsivity: an Existential-Phenomenological Study of Consumers with Attention Deficit Disorder. Psychology \& Marketing, v. 21(8), p. 637-669, 2004.

KEENING, Peter; PLASSMANN, Hilke; AHLERT, Dieter. Applications of Functional Magnetic Resonance Imaging For Market Research. Qualitative Market Research, v. 10 (2), p. 135-152, 2007.

KEMP, Elyria; BUI, My; GRIER, Sonya. Eating Their Feelings: Examining Emotional Eating in At-Risk Groups in the United States. Journal of Consumer Policy, v. 34, p. 211-229, 2011.

KENNING, Peter; LINZMAJER, Marc. Consumer Neuroscience: an Overview of an Emerging Discipline with Implications for Consumer Policy. Journal für Verbraucherschutz und Lebensmittelsicherheit, v. 6, p. 111-125, 2011.

KOLLER, Monika. Special Issue on "Neuromarketing". Editorial. Der Markt Journal für Marketing, v. 49, p. 127-128, 2010.

LEE, Nick; BRODERICK, Amanda J.; CHAMBERLAIN, Laura. What is 'Neuromarketing'? a Discussion and Agenda for Future Research. International Journal of Psychophysiology, v. 63, p. 199-204, 2007.

MALHOTRA, Naresh K. Pesquisa de marketing: uma orientação aplicada. 6. ed. Porto Alegre: Bookman, 2008.

McELROY, Susan L. et al. Compulsive Buying: a Report of 20 Cases. Journal of Clinical Psychiatry, v. 55 (6), p. 242-248, 1994. 
MISHRA, Arul; MISHRA, Himanshu. We are what We Consume: the Influence of Food Consumption on Impulsive Choice. Journal of Marketing Research, v. 47, p. 1129-1137, December 2010.

MLODINOW, Leonard. Subliminar: como o inconsciente influencia nossas vidas. Rio de Janeiro: Zahar, 2012.

MORIN, Christophe. Neuromarketing: the New Science of Consumer Behavior. Society, v. 48 (2), p. 131-135, 2011.

MURPHY, Emily; ILLES, Judy; REINER, Peter. Neuroethics of Neuromarketing. Journal of Consumer Behaviour, v. 7, p. 293-302, July-October, 2008

O'GUINN, T.C. and FABER, R.J. Compulsive Buying: a Phenomenological Exploration. Journal of Consumer Research, v. 16 (2), p. 147-57, 1989.

OLIVEIRA, Jorge; GIRARDI, Janaina; SANTOS, Rene. Opening the "Black Box" in the Consumer's Mind: Understanding what is Neuromarketing. International Journal of Business and Management, vol. 9 (9), p. 96-107, 2014.

OTTAVIANI, Cristina; VANDONE, Daniela. Impulsivity and Household Indebtedness: Evidence from Real Life. Journal of Economic Psychology, v. 32 (5), p. 754-761, 2011.

PAYZAN, Elise; BOURGEOIS-GIRONDE, Sacha. Epistemological Foundations for Neuroeconomics. HAL, Working Paper, 2005.

PERRACHIONE, Tyler K.; PERRACHIONE, John R. Brains and Brands: Developing Mutually Informative Research in Neuroscience and Marketing. Journal of Consumer Behaviour, v. 7, p. 303-318, 2008.

PLASSMANN, Hilke; RAMSOY, Thomas Zoëga; MILOSAVLJEVIC, Milica. Branding the Brain: a Critical Review and Outlook. Journal of Consumer Psychology, v. 22, p. 18-36, 2012.

REIMANN, M. et al. Functional Magnetic Resonance Imaging in Consumer Research: a Review and Application. Psychology and Marketing (28)6, p. 608-637, 2011.

ROOK, Dennis W.; FISCHER, Robert J. Normative Influences on Impulsive Buying Behavior. Journal of Consumer Research, v. 22 (3), p. 305-313, 1995.

SANTINI, Fernando. Uma análise da influência da promoção de vendas de desconto na intenção de compra do consumidor e os efeitos moderadores da atratividade. 2013. $199 \mathrm{f}$. Tese (Doutorado em Administração) - Programa de Pós-Graduação em Administração, PPGAd/PUCRS, Porto Alegre, 2013. 
SANTOS, Marcos; GONÇALVES, Carlos; MONTEIRO, Plínio; GONÇALVES FILHO, Cid. Refletindo sobre a ética na prática do neuromarketing: a neuroética. REMark - Revista Brasileira de Marketing, v. 13 (3), p. 49-62, 2014.

SEIDERS, K.; PETTY, R. D. Obesity and the Role of Food Marketing: a Policy Analysis of Issues and Remedies. Journal of Public Policy and Marketing, v. 23, p. 153-169, Fall, 2004.

SENIOR, Carl; SMYTH, Hannah; COOKE, Richard; SHAW, Rachel L.; PEEL, Elizabeth. Mapping the Brain for the Modern Market Researcher. Qualitative Market Research, v. 10 (2), p. 153-167, 2007.

SENIOR, Carl; LEE, Nick. A Manifesto for Neuromarketing Science (Editorial). Journal of Consumer Behaviour, v. 7, p. 263-271, July-October, 2008.

SHIV, B. et al. Decision Neuroscience. Marketing Letters 16:3/4, p. 375-386, 2005.

SILVERA, David H.; LAVACK, Anne M.; KROPP, Fredric. Impulse Buying: the Role of Affect, Social Influence, and Subjective Wellbeing. Journal of Consumer Marketing, v. 25 (1), p. 23-33, 2008.

SNEATH, Julie Z.; LACEY, Russell; KENNETT-HENSEL, Pamela A. Coping with a Natural Disaster: Losses, Emotions and Impulsive and Compulsive Buying. Marketing Letters, v. 10 (20), p. 45-60, 2009.

SOLNAIS, Céline; AUDREU-PEREZ, Javier; SÁNCHEZ-FERNÁNDEZ, Juan; ANDRÉUABELA, Jaime. The Contribution of Neuroscience to Consumer Research: A Conceptual Framework and Empirical Review. Journal of Economic Psychology, v. 36, p. 68-81, 2013.

TURINETTI, Erin; ZHUANG, Hong. Exploring Determinants of U.S. Household Debt. Journal of Applied Business Research, v. 27 (6), p. 85-92, 2011.

VENKATRAMAN, Vinod; CLITHERO, John A.; FITZSIMONS, Gavan J.; HUETTEL, Scott A. New Scanner Data for Brand Marketers: How Neuroscience Can Help Better Understand Differences in Brand Preferences. Journal of Consumer Psychology, v. 22, p. 143-153, 2012.

VENKATRAMAN, V; PAYNE, J.W. Opening the blackbox: Process Tracing in Decision Research. In: SCHULTE-MECKLENBECK, M.; KÜHBERGER, A.; RANYARD, R. (Eds.). Handbook of Process Tracing Methods in Decision Making, London: Psychology Press, 2011.

WALTER, Henrik; ABLER, Birgit; CIARAMIDARO, Angela; ERK, Susanne. Motivating Forces of Human Actions: Neuroimaging Reward and Social Interaction. Brain Research Bulletin, v. 67, p. 368-381, 2005. 
WEUN, Seungoog; JONES, Michael A.; BEATTY, Sharon E. The Development and Validation of the Impulse Buying Tendency Scale. Psychological Reports, v. 82, p 1123$1133,1998$.

WORLD MEDICAL ASSOCIATION. Medical Ethics Manual, $2^{\text {nd }}$ Ed. 2009. 140p.

YOON, C. et al. Decision Neuroscience and Consumer Decision Making. Marketing Letters. 23, p. 473-485, 2012. 